\title{
Pharmacological effects of medicinal components of Atractylodes lancea (Thunb.) DC.
}

\author{
Xie Jun ${ }^{1 \dagger}$, Peng Fu ${ }^{2+}$, Yu Lei ${ }^{1}$ and Peng Cheng ${ }^{1 *}$
}

\begin{abstract}
Atractylodes lancea Thunb. DC. (AL) has a long history as one of the important herbs used in East Asia. This review is on the purpose of providing a comprehensive summary of the pharmacological effects of AL and its extractions. The publication from PubMed, ScienceDirect, Springer, and Wiley database was collected and summarized. The potential application of AL on the disease could be attributed to its pharmacological properties such as anti-cancer, anti-inflammatory and other essential effects. Hence, this review aims at providing evidence of the pharmacological activities of $\mathrm{AL}$ as one of natural products used in clinical trial.
\end{abstract}

Keywords: Natural product, AL, Pharmacological activities

\section{Background}

Atractylodes lancea Thunb. DC. (AL) has a long history as one of the important herbs used in East Asia. AL is grown mainly in Hubei and Jiangsu. In traditional Chinese medicine (TCM), the rhizome of AL tastes pungent and bitter, and belongs to spleen, stomach and liver meridian. AL could eliminate dampness, strengthen the spleen, eliminating wind and dispersing cold. According to TCM theory, AL was traditionally used to treat rheumatic diseases, digestive disorders, night blindness, and influenza [1]. AL was generally applied into traditional decoction, such as Ermiao Powder, Simiao Powder, Yueju-Wan and several other famous decoctions. Recently, it was interesting to find the extract from AL could also exert anticancer, anti-obesity and anti-inflammatory effects [2]. AL contains sesquiterpenes, sesquiterpenoids, polyethylene alkynes, phytosterols and etc., such as elemol, $\beta$-selinene and atractylone $[3,4]$. Sesquiterpenes are a class of natural products composed of three isoprene units, derived from the 15-carbon farnesyl pyrophosphate. Sesquiterpenoids are extensively distributed in nature with 15-carbon after biochemical modifications of sesquiterpenes. Alkynes are a class of organic compounds composed of

\footnotetext{
*Correspondence: pengchengchengdu@126.com

${ }^{+}$Xie Jun and Peng Fu contributed equally to this work

${ }^{1}$ Chengdu University of Traditional Chinese Medicine, Chengdu, China

Full list of author information is available at the end of the article
}

at least one carbon-carbon triple bond, easily undergoing oxidative cleavage. Phytosterols are a subgroup of the steroids. Steroids are a class of organic molecules composed of seventeen carbon atoms, with four rings arranged in a specific molecular configuration. Especially, this review article will concentrate on the major constituents identified in AL with multi-pharmacological activities $[5,6]$. The names are shown in Table 1 and structures are shown in Fig. 1.

\section{Pharmacological effects \\ Atractylodes lancea Thunb. DC.}

Opisthorchis viverrini (OV)-induced cholangiocarcinoma (CCA) is the common cancer between male and female, especially in Thailand. Recent study exhibited that the ethanol extract of AL exerted an inhibitory effect on OV-induced CCA animal model without obvious toxicity through oral administration for 30 days according to the results from positron emission tomography-computed tomography and male Syrian hamsters [7]. In order to explore the effect of AL rhizome on gastrointestinal motility, the atropine-, dopamine-, and 5-hydroxytryptamine (5-HT)-treated mice were adopted in the study. The results exerted that $1 \mathrm{mg} / \mathrm{kg}$ extract could vitally suppress dopamineinduced gastric emptying and gastrointestinal motility decrease through intraperitoneal injection. The extract at the concentration of $1000 \mathrm{mg} / \mathrm{kg}$ could restrict 
Table 1 The chemical information of major components of $A L$

\begin{tabular}{llll}
\hline Chemical name & Formula & Type of compound & $\begin{array}{l}\text { Molecular } \\
\text { weight } \mathbf{( g /} \\
\text { mol) }\end{array}$ \\
\hline Atractylenolide I & $\mathrm{C}_{15} \mathrm{H}_{18} \mathrm{O}_{2}$ & Sesquiterpene & 230.30 \\
Atractylenolidell & $\mathrm{C}_{15} \mathrm{H}_{20} \mathrm{O}_{2}$ & Sesquiterpene & 232.32 \\
Atractylenolide III & $\mathrm{C}_{15} \mathrm{H}_{20} \mathrm{O}_{3}$ & Sesquiterpene & 248.32 \\
Atractylone & $\mathrm{C}_{15} \mathrm{H}_{20} \mathrm{O}$ & Sesquiterpenoid & 216.32 \\
Hinesol & $\mathrm{C}_{15} \mathrm{H}_{26} \mathrm{O}$ & Sesquiterpenoid & 216.3 \\
B-Eudesmol & $\mathrm{C}_{15} \mathrm{H}_{26} \mathrm{O}$ & Sesquiterpenoid & 222.37 \\
Atractylodin & $\mathrm{C}_{13} \mathrm{H}_{10} \mathrm{O}$ & Polyethylene alkyne & 182.22 \\
Stigmasterol & $\mathrm{C}_{29} \mathrm{H}_{48} \mathrm{O}$ & Phytosterol & 412.69 \\
$\beta$-Sitosterol & $\mathrm{C}_{29} \mathrm{H}_{50} \mathrm{O}$ & Phytosterol & 414.71 \\
\hline
\end{tabular}

atropine-induced small intestinal motility decrease, and the extract of $500 \mathrm{mg} / \mathrm{kg}$ dose could block 5 -HTinduced gastric emptying and small intestinal motility decrease [8]. Additionally, AL exerted anti-gastric ulcer effect in the rat model through decreasing inflammatory mediators (i.e. tumor necrosis factor- $\alpha$ (TNF$\alpha$ ), interleukin-8 (IL-8), IL-6), and prostaglandin $E_{2}$ $\left(\mathrm{PGE}_{2}\right)$ and increasing epidermal growth factor (EGF) and trefoil factor2 (TFF2) [9]. Soil-borne fungal pathogens reduced $30 \%$ yields of peanuts [10]. Recently, it was reported that intercropping with the peanuts, AL could also suppress soil-borne fungal diseases through the inhibition of Fusarium oxysporum growth [11]. Petroleum ether (PE) extraction from AL showed an inhibitory effect on BGC-823 and SGC-7901 human gastric cancer cells. PE fraction could induce apoptosis, cell cycle arrest, and mitochondrial transmembrane potential changes [12]. A recent report exerted that $\mathrm{MeOH}$ extract of $\mathrm{AL}$ could inhibit nitric oxide (NO) production in lipopolysaccharide-stimulated murine macrophage-like RAW264.7 cells and induce granulocyte-colony stimulating factor (G-CSF) secretion in murine normal colonic epithelial MCE301 cells. NO is a free radical [13]. The ethanol extract of $\mathrm{AL}$ exerted an essential inhibitory effect on lipase with the $\mathrm{IC}_{50} 9.06 \mu \mathrm{g} / \mathrm{mL}$ in a human pancreatic lipase assay, and exhibited anti-obesity effect on a high-fat dietinduced obesity mice model at the high concentration of $500 \mathrm{mg} / \mathrm{kg}$ [14]. The water extracts from AL showed the antagonistic activity against serotonin receptors and an inhibitory effect on the selective 5-HT2A/2C agonist 1-(2,5-dimethoxy-4-iodophenyl)-2-aminopropane-induced head-twitch response in mice [15]. Water extracts from AL reduced triptolide-induced toxicity through the down-regulation of the hepatic expression of CYP3A. In the meantime, AL could improve anti-inflammatory effects of triptolide. These findings suggested that AL could inhibit toxicity and increase therapeutic activity of triptolide through combination [16].

\section{Atractylenolide I}

Atractylenolide I (ATL-I), a sesquiterpene, can be extracted from Rhizoma Atractylodis Macrocephalae (RAM) and AL [17]. ATL-I is soluble in Ethanol and Methanol, and is stable without exposure to light or moisture. ATL-I exerted multiple therapeutic activities, such as anti-cancer, anti-inflammatory and anti-atopic effects [18-20]. ATL-I was identified in AL as one of the main components in 2009 [21]. Bladder cancer ranks the second most common urological worldwide [22]. ATL-I recently was demonstrated to have the anti-tumor effect on bladder cancer in vivo and in vitro. It was found that ATL-I increased the p21 level and decreased the level of cyclin B1, CDK1 and Cdc25c, resulting in the inhibition of bladder cancer cell proliferation. Further study showed that ATL-I could induce cell cycle arrest in G2/M phase. Additionally, ATL-I had a stimulatory effect on apoptosis through the suppression of PI3 K/Akt/mTOR signaling pathway. The animal study showed that ATL-I could inhibit tumor growth without the obvious toxicity [17]. Leukemia refers to a type of cancers generally starting from the bone marrow and resulting in abnormal white blood cells. Recent studies showed that ATL-I could inhibit AML and CML leukemia cell proliferation and growth. MTT assay showed that ATL-I could inhibit K562 CML and U937 AML. ELISA and IF assay exhibited that ATL-I could induce apoptosis. Additionally, ATL-I also promoted caspase- 3 and caspase- 9 activation through the cleavage of pro-caspase- 3 and pro-caspase- 9 . Caspase- 3 and caspase- 9 are an enzymes initiating apoptotic pathway. Also, ATL-I could increase the CD14 and CD68 markers, leading to the cell differentiation of ATLI-treated leukemia cells [23]. Melanoma belongs to the skin cancer [24]. Recent researches showed that ATL-I could inhibit human melanoma cells growth through the induction of apoptosis. Further study exerted that ATL-I could suppress melanoma cell migration through the inhibition on p-JAK2, p-STAT3, MMP-2 and MMP9. MMP-2 and MMP-9 are matrix metallopeptidases involved in invasion and metastasis in multiple cancers. Interestingly, the overexpression of STAT3 would at least partly reverse the anti-cancer effect of ATL-I on melanoma cells [25]. The clinical study about the evaluation of therapeutic effect of ATL-I on gastric cancer cachexia patients has been conducted in recent years. According to the results about appetite, body weight, mid-arm muscle circumference, Karnofsky performance status (KPS) status, ATL-I could improve appetite and KPS 
<smiles>C=C1CCC[C@]2(C)C=C3OC(=O)C(C)=C3C[C@]12C</smiles>

\section{Atractylenolide I}<smiles>C=C1CCC[C@]2(C)Cc3occ(C)c3C[C@H]12</smiles>

Atractylone<smiles>C=C1CCC[C@]2(C)C[C@]3(C)OC(=O)C(C)=C3C[C@]12C</smiles>

Atractylenolide II<smiles>CC1=CCCC(C)C12CC[C@@H](C(C)(C)O)C2</smiles>

Hinesol<smiles>C=C1CCC[C@]2(C)C[C@]3(O)OC(=O)C(C)=C3C[C@]12C</smiles>

Atractylenolide III<smiles>C=C1CCC[C@]2(C)CC[C@H](C(C)(C)O)C[C@@H]12</smiles>

$\beta$-eudesmol

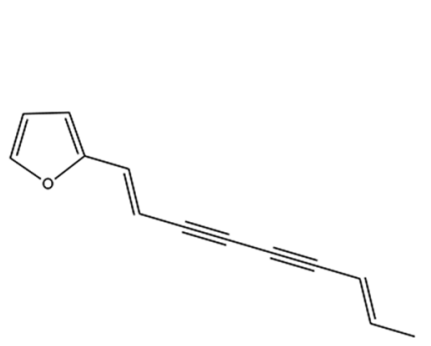

Atractylodin

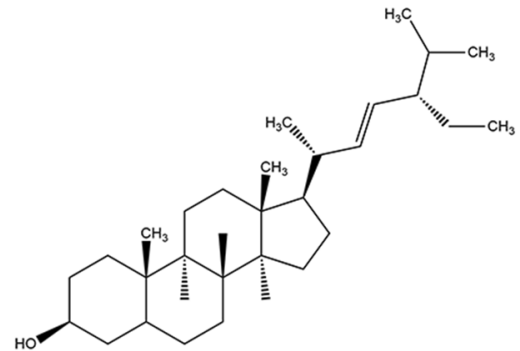

Stigmasterol

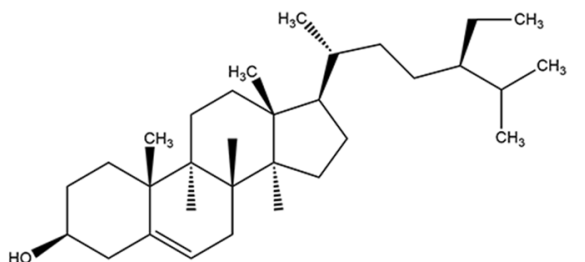

$\beta$-sitosterol

Fig. 1 The chemical structures of pure compounds extracted from AL (drawing via ChemDraw)

status. Meanwhile, the IHC staining results showed that ATL-I could increase TNF- $\alpha$ expression and reduce IL-1 and proteolysis-inducing factor (PIF) production [26]. Intestinal epithelial (IEC-6) cells showed an important role in gastrointestinal disease with the ability to promote and accelerate the healing of mucosal ulcers and wounds. Recent study found that ATL-I could promote IEC- 6 cell migration and proliferation, with an increase of cytosolic free $\mathrm{Ca}^{2+}$ concentration $\left(\mathrm{Ca}^{2+}\right)$. Further study exerted that ATL-I could increase TRPC1 and PLC- ${ }_{\gamma 1}$ in IEC-6 cells [27]. Moreover, ATL-I suppressed vascular smooth muscle cell proliferation, migration, lipid peroxidation and inflammatory responses induced by oxidized modified low-density lipoprotein [28]. A present research indicated that ATL-I had a protective effect on the acute lung injury in the lipopolysaccharide (LPS)-induced mice. The detailed mechanism was associated with suppression of toll like receptor 4 (TLR4) expression and NF- $\mathrm{kB}$ activation. NF- $\mathrm{kB}$ could promote the transcription of oncogenic genes, cytokine production and cell survival after inducers stimulation, such as ROS, TNF $\alpha$ and IL- $1 \beta$. It was found that ATL-I powerfully suppressed lung wet-to-dry weight ratio. Simultaneously, attenuating the LPS-induced pathological changes in lung tissues included inflammatory cells infiltration, interalveolar septal thickening, and edema. The further 
study showed that treatment with ATL-I considerably down-regulated the release of TNF- $\alpha$, IL- 6 , IL- $1 \beta$, and IL-13, which were detected by ELISA. However, ATL-I could enhance the expression of IL-10 in BALF. LPS could induce conformational changes in the TLR4 receptors to activate TLR4. TLR4 activation would promote NF- $\mathrm{kB}$ expression and inflammatory cytokine production. Additionally, ATL-I also had an inhibitory effect on the LPS-induced TLR4 expression and NF- $\mathrm{BB}$ activation, which were measured by western blot analysis [29]. It was interesting that recent study showed that ATL-I could inhibit uridine $5^{\prime}$-iphospho-glucuronosyltransferases (UGTs) isoforms, especially UGT2B7 [30]. Additionally, ATL-I could inhibit 5-LOX production to decrease allergic response [31].

\section{Atractylenolide II}

Atractylenolide II (ATL-II), a sesquiterpene, can be extracted from RAM and AL, exerting anti-cancer and anti-inflammatory activities. The melting point of ATLII is $383{ }^{\circ} \mathrm{C}$. ATL-II was identified in AL in 1998 [32]. Recent study showed that suppression of STAT3 signaling had a promoting effect on the anti-melanoma action of AT-II in B16 and A375 cells. 48 h AT-II treatment showed a dose-dependent manner inhibitory effect on p-STAT3, p-Src, and Mcl-1 and Bcl-xL in B16 and A375 cells. However, the suppressive effect of AT-II would be mostly reversed after the restoration of active variant of STAT3. The animal studies showed that 14-day administration of AT-II could obviously suppress tumor growth and inhibit STAT3 activation [33]. A present study manifested that AT-II could significantly inhibit human gastric carcinoma cell HGC-27 and AGS growth and migration in a dose-dependent manner. Further study showed that AT-II could suppress Bcl-2, p-Akt and p-ERK expression, and increase Bax expression [34].

\section{Atractylenolide III}

Atractylenolide III (ATL-III), a sesquiterpene, is the major component in RAM, and could be extracted from $\mathrm{AL}$. The melting point of ATL-III is $200-201{ }^{\circ} \mathrm{C}$, and the freezing point of ATL-III is $392-394{ }^{\circ} \mathrm{F}$. ATL-III showed the cytotoxic effect on cancer cells and the anti-inflammatory effect on macrophages [35, 36]. ATL-III was identified in AL in 2008 [37]. Mast cells could promote atopic dermatitis [38]. It was demonstrated that ATL-III possessed anti-tumor activity on TSLP-stimulated human mast cell, HMC-1 cells. The detailed mechanism was that ATL-III could inhibit the murine double minute 2 levels, mast cell proliferation, IL-13 and phosphorylated signal transducer and activator of transcription. In the mean time, ATL-III could promote p53 expression. Additionally, the release of pro-inflammatory cytokines stimulated by TSLP (IL-6, TNF- $\alpha$, and IL-8) was reduced by ATLIII. Furthermore, the Bcl2 and pro-caspase-3 levels could be down regulated by ATL-III, whereas the caspase- 3 activation and cleaved PARP levels could be up regulated by ATL-III [39]. Recent study showed that ATLIII exhibited anti-inflammatory effect on LPS-triggered RAW264.7 mouse macrophages. The detailed mechanism was associated with the inhibitory effect of ATL-III on the production of NO, PGE2, TNF- $\alpha$ and IL-6, and the suppressive effect of ATL-III on NF- $\kappa B$ and mitogenactivated protein kinase (MAPK) signaling pathways. ATL-III could dose-dependently inhibit NO, TNF- $\alpha$, PGE2 and IL-6 release, and high concentration of ATLIII $(100 \mu \mathrm{M})$ could suppress cytokines cyclooxygenase-2 (COX-2) expression via the inhibition on the activation of NF-kB and ERK1/2 [40]. Additionally, ATL-III exerted a neuroprotective effect in rats with learning and memory impairment through the inhibition of ROS production and protein kinase $C$ levels [41].

\section{Atractylone}

Atractylone was one of the main sesquiterpenic constituents of AL, displaying the anti-inflammatory action and anti-hepatotoxic effect [42-44]. The boiling point is $285.00-286.00{ }^{\circ} \mathrm{C}$. Atractylone was identified in AL in 2006, reaching 9.35\% [45]. Atractylone showed an inhibitory effect on allergic inflammation in an ovalbumin (OVA)-induced AR animal model. Further study exerted that Atractylone could also suppress caspase-1/NF- $\mathrm{kB} /$ MAPKs activation phorbol 12-myristate 13-acetate and calcium ionophore A23187 (PMACI)-induced human mast cell line (HMC-1) cells. In the animal model, Atractylone decreased rub scores, and reduced IL-1, IL-4, IL-5, IL-6, IL-13, COX-2, intercellular adhesion molecule-1, and macrophage inflammatory protein-2 expression [46]. Recently, atractylone could decrease influenza A virus (IAV)-induced pulmonary injury at the concentration of $40 \mathrm{mg} / \mathrm{kg}$ for 5 days in mice model. Further study showed that atractylone could inhibit IL-6, TNF- $\alpha$ and IL-1 $\beta$, but promote Toll-like receptor 7 (TLR7), MyD88, tumor necrosis factor receptor-associated factor 6 interferon- $\beta$ (IFN- $\beta$ ) expression [47]. It was reported that atractylone exerted a salutary effect on the mast cellmediated allergic reactions. The results exhibited that atractylone could suppress compound 48/80-induced rat peritoneal mast cells (RPMCs) degranulation intracellular $\mathrm{Ca}^{2+}$ level $\left(\left[\mathrm{Ca}^{2+}\right]\right)$, tryptase release, and histamine release. Additionally, atractylone could inhibit compound 48/80-induced p56lck tyrosine kinase activity in RPMCs. Also, atractylone showed an inhibitory effect on histidine decarboxylase activity and expression in PMACI-induced HMC-1 cells. Further study showed that atractylone could also inhibit tryptase and histamine releases in 
PMACI-induced HMC-1 cells. Atractylone could inhibit morphological alteration and filamentous actin formation in stem cell factor-stimulated RPMCs animal model [48].

\section{Hinesol}

Hinesol is a sesquiterpenoid, which was $5-9 \%$ in AL. Hinesol exhibited the cytotoxic effect on cancer cells and a strong anti-inflammatory effect. Hinesol was clarified in AL in 2003 [49]. Hinesol could reduce nuclear fragmentation and DNA fragmentation, indicating that hinesol had an inhibitory effect on human leukemia HL-60 cells through apoptosis. The further study showed that hinesol could modulate c-Jun signaling pathway through the activation of c-Jun N-terminal kinase (JNK) [50]. A recent study indicated that hinesol could show the anti-gastric ulcer effect through a significant inhibitory effect on $\mathrm{H}^{+}, \mathrm{K}^{+}$-ATPase activity. The concentration of ATP and $\mathrm{K}^{+}$could not modulate the inhibitory effect of hinesol, but the increase of $\mathrm{Mg}^{2+}$ concentration could promote the inhibitory effect of hinesol. Also, hinesol displayed a moderate inhibitory effect on $\mathrm{Mg}^{2+}$-ATPase and $\mathrm{Ca}^{2+}$-ATPase activity [51].

\section{$\beta$-Eudesmol}

$\beta$-Eudesmol, a sesquiterpenoid alcohol, is the main constituent of AL. $\beta$-Eudesmol can be also extracted from Teucrium ramosissimum [52]. The melting point of $\beta$-eudesmol is $72-74{ }^{\circ} \mathrm{C}$. Particularly, $\beta$-eudesmol demonstrated a strong inhibitory effect on cancer, and $\beta$-eudesmol could protect neuron from inflammatory damage. CCA, or bile duct cancer, which was an uncommon adenocarcinoma that originated from the epithelial cells of bile ducts, is becoming a vital public health problem worldwide. Recent study exerted that $\beta$-eudesmol could improve the anti-cancer effect of 5-fluorouracil and doxorubicin in human CCA KKU-100 cells with the high expression of NAD (P) H-quinone oxidoreductase 1 (NQO1). Also, $\beta$-eudesmol could inhibit cell growth, migration, NQO1 expression and activity in CCA [53]. $\beta$-Eudesmol showed an inhibitory effect on CCA growth and metastasis in CCA-xenografted nude mouse model according to the results from positron emission tomography-computed tomography (PET-CT). Also, $\beta$-eudesmol could prolong the survival time of CCA mice model [54]. Another study exerted that $\beta$-eudesmol could also induce apoptosis, cell cycle arrest at G1 phase, and the cleavage of caspase 3 and caspase 7 in CCA [55]. Primary liver cancer is the third-leading cause of cancer death all over the world [56]. Recent studies showed that $\beta$-eudesmol isomers could inhibit proliferation of human hepatocellular carcinoma Hep-G2 cells through the induction of apoptosis, according to haematoxylin-eosin and acridine orange ethidium bromide staining results. Further study showed that $\beta$-eudesmol could decrease mitochondrial membrane potential and activate caspases [57]. Recently, $\beta$-eudesmol showed the anti-tumor activities in human lung and colon cancer cells. Further study exerted that $\beta$-eudesmol $(200 \mu \mathrm{M})$ significantly decreased A549 cells migration towards type IV collagen (54\% inhibition) and fibronectin ( $60 \%$ inhibition). Meanwhile, $\beta$-eudesmol $(200 \mu \mathrm{M})$ also inhibited HT29 cell migration toward type IV collagen and fibronectinline with inhibition of $76 \%$ and $63 \%$, respectively [52]. Abnormal angiogenesis was involved in diverse diseases, such as tumor and diabetic retinopathy. Recently, $\beta$-eudesmol showed an inhibitory effect on angiogenesis partly via the arrest of the ERK signaling pathway, suggesting that $\beta$-eudesmol could be considered as the drug candidate for treatment of angiogenic diseases. In detail, $\beta$-eudesmol had an inhibitory effect on the multiplication of porcine brain microvascular endothelial cells and human umbilical vein endothelial cells (HUVEC). Additionally, $\beta$-eudesmol could inhibit the migration of HUVEC, and at the high concentration $\beta$-eudesmol could inhibit the ERK1/2 phosphorylation and suppress angiogenesis in subcutaneously implanted Matrigel plugs in mice and in adjuvantinduced granuloma in mice [58]. Another study showed that $\beta$-eudesmol had an inhibitory effect on angiogenesis via inhibiting CREB activation in growth factor signaling pathway, herein demonstrating $\beta$-eudesmol as an inhibitory compound of tumor growth. $\beta$-Eudesmol at the concentration ranging from 50 to $100 \mu \mathrm{M}$ had an inhibitory effect on the proliferation of HUVEC stimulated with vascular endothelial growth factor (VEGF, $30 \mathrm{ng} / \mathrm{ml}$ ) and basic fibroblast growth factor (bFGF, $30 \mathrm{ng} / \mathrm{ml}$ ). In addition, $\beta$-eudesmol at the concentration of $100 \mu \mathrm{M}$ also had a blocking effect on the phosphorylation of cAMP response element binding protein (CREB) triggered by VEGF $30 \mathrm{ng} / \mathrm{ml}$ in HUVEC. $\beta$-Eudesmol at the concentration ranging from 10 to $100 \mu \mathrm{M}$ suppressed proliferation of HeLa, SGC-7901, and BEL-7402 tumor cells in a time-and dose-dependent manner. Moreover, treatment with $\beta$-eudesmol $(2.5-5 \mathrm{mg} / \mathrm{kg})$ markedly suppressed the growth of $\mathrm{H} 22$ and $\mathrm{S} 180$ mouse tumor growth in vivo [59]. Pheochromocytoma mainly refers to a neuroendocrine tumor of the adrenal glands. $\beta$-Eudesmol could induce neurite extension in rat pheochromocytoma cells (PC-12), accompanying with inhibition of $\left[{ }^{3} \mathrm{H}\right]$ thymidine incorporation. Meanwhile, $\beta$-eudesmol also stimulated the improvement of $\left[\mathrm{Ca}^{2+}\right]$. Moreover, $\beta$-eudesmol concentration-dependently led to an accumulation of inositol phosphates, and $\beta$-eudesmol could increase the p-MAPK time-dependently [60]. Recently, $\beta$-eudesmol was reported to modify the sensitivity of diabetic mice to depolarizing blockers so as to increase 
the susceptibility to these compounds. By investigating phrenic nerve-diaphragm muscles in normal and alloxandiabetic mice, $\beta$-eudesmol exhibited the potentiating/ promoting effect on neuromuscular blockade. Pretreatment with $\beta$-eudesmol enhanced the blocking action of succinylcholine to a greater degree in diabetic muscles than in normal ones. 30-min pretreatment in normal muscles could make the effect saturated,while 60-min pretreatment in diabetic muscles could make further potentiation [61]. It was reported that $\beta$-eudesmol could block the nicotinic acetylcholine receptor (nAChR) channel in both open and closed conformations, resulting in potentiating the neuromuscular blockade induced by succinylcholine (SuCh). The blocking effect of SuCh $(0.1-10 \mu \mathrm{M})$ with $\beta$-eudesmol on nAChR channel activity was investigated by using the cell-attached patch clamp technique. Pretreatment with $\beta$-eudesmol $(20 \mu \mathrm{M})$ had no effect on resting membrane potential and AChactivated channel activities. $\beta$-Eudesmol decreased $\mathrm{SuCh}$ (above $0.3 \mu \mathrm{M}$ )-induced prolongation of channel open time and reduced the frequency of channel opening in the presence of SuCh (above $3 \mu \mathrm{M}$ ) in ACh-activated channel currents regulated by SuCh [62]. $\beta$-Eudesmol could potentiate the effect of phenylene-polymethylenebis-ammonium (PMBA) derivatives on neuromuscular blockades in alloxan-diabetic mice [63]. Current study manifested that the intragastric injection of $\beta$-eudesmol to rats could inhibit efferent adrenal sympathetic nerve activity (ASNA). The knock-out of TRPA1 could block the inhibitory effect of $\beta$-eudesmol on ASNA, and subdiaphragmatic vagotomy could promote the suppression of $\beta$-eudesmol on ASNA, suggesting that $\beta$-eudesmol could modulate ASNA through TRPA1 and afferent vagus nerve [7]. It was reported that $\beta$-eudesmol had the ability of modifying the chemical composition of the workers cuticle, to impair nestmate recognition, initiate alarm behavior and result in nestmate aggression [64]. Recent study showed that $\beta$-eudesmol pre-treatment on mouse diaphragm muscles for $30-60$ min could promote $\left[\mathrm{Ca}^{2+}\right]$ and twitch tension induced by succinylcholine as nicotinic acetylcholine receptor channel [65].

\section{Atractylodin}

Atractylodin (Atr), a polyethylene alkyne, could be extracted from AL, exerting the anti-inflammatory activities. The melting point of Atr is $52{ }^{\circ} \mathrm{C}$. A present study showed that atractylodin (Atr) could inhibit LPS-induced inflammatory responses. Namely, Atr could suppress myeloperoxidase (MPO) activity, the wet-to-dry weight ratio of the lungs, protein leakage, infiltration of inflammatory cells, TNF- $\alpha$, IL- 6 , IL- $1 \beta$ and monocyte chemoattractant protein (MCP)-1 secretion. The mechanism was that Atr could down-regulate nucleotide-binding domain- (NOD-) like receptor protein 3 (NLRP3) inflammasome and TLR4 activation [66]. Another study reported that Atr exhibited anti-inflammatory effects and ameliorated concurring dysmotility in constipationprominent $(\mathrm{CP})$ and diarrheaprominent (DP) rats. The detailed mechanism was associated with the reduction of the plasma pro-inflammatory cytokines such as TNF- $\alpha$, IL-1 $\beta$, and IL-6 [67].

\section{Stigmasterol}

Stigmasterol is an unsaturated phytosterol, and could extracted from soybean, calabar bean, rape seed, American Ginseng, AL and etc. The melting point of stigmasterol is $160-164{ }^{\circ} \mathrm{C}$. Stigmasterol showed diverse pharmacological effects, such as anti-inflammotory, anti-nociceptive and anti-diabetic activities. Recent study exerted that stigmasterol could inhibit OVA-induced airway inflammatory damage in guinea pigs through intraperitoneal injection. More precisely, stigmasterol could suppress eosinophils, lymphocytes, and monocytes proliferation and inhibit peribronchiolar, perivascular, and alveolar infiltration of inflammatory cells. Stigmasterol could also inhibit vascular cell adhesion molecule-1 (VCAM-1) and OVA-specific immunoglobulin E (OVA sIgE) expression. VCAM-1 could medicate the adhesion of lymphocytes, monocytes, eosinophils, and basophils to vascular endothelium after the endothelial cells are stimulated by cytokines [68]. Recent study showed stigmasterol could improve memory and behavioral impairments in vanadium-induced neurotoxicity. More precisely, stigmasterol could reduce escape latency and prolong swimming time in Morris water maze. Also, stigmasterol could promote activities of antioxidant enzymes, and inhibit oxidative stress markers as well as lipid peroxidation in mice hippocampal homogenates [69]. A recent study showed that stigmasterol could protect mice from LPS-induced fever response. Further study exerted that stigmasterol relived organ damage and dearth rate [70]. Recently, stigmasterol exerted the synergistic antibiotic effect as adjuvant of ampicillin against both Gram positive and Gram-negative bacteria from clinical isolates. The inhibitory effect of ampicillin and stigmasterol alone is extremely ineffective, but the combination of stigmasterol-ampicillin significantly suppress colony counts, yielding 98.7\% [71]. Trypanosoma congolense could induce the disease nagan in diverse animals. Stigmasterol showed antitrypanosomal activity against Trypanosoma congolense infection through reducing sialidase [72]. Moreover, a present study exhibited that stigmasterol displayed a mosquito larvicidal activity through neurotoxicity [73]. A present study displayed that stigmasterol showed anti-nociceptive effect on male albino Swiss mice model according to the results from 
Table 2 The anti-cancer effects of major components of AL

\begin{tabular}{|c|c|c|c|c|}
\hline Chemical name & Tumor suppressive effect & Potential targets & Function study & References \\
\hline \multirow[t]{3}{*}{ Atractylenolide I } & Anti-bladder cancer effect & $\downarrow \mathrm{PISK} / \mathrm{Akt} / \mathrm{mTOR}$ & In vitro and In vivo & [17] \\
\hline & Anti-leukemia effect & $\uparrow C D 14 / C D 68$ & In vitro & [23] \\
\hline & Anti-melanoma effect & $\downarrow p-J A K 2 / p-S T A T 3$ & In vitro & [25] \\
\hline \multirow[t]{2}{*}{ Atractylenolide II } & Anti-melanoma effect & $\downarrow p-S T A T 3 / p-S r c$ & In vitro and in vivo & [33] \\
\hline & Anti-gastric cancer effect & $\downarrow p-A k t / p-E R K$ & In vitro & [34] \\
\hline Hinesol & Anti-leukemia effect & $\downarrow c$-Jun & In vitro & [50] \\
\hline$\beta$-Eudesmol & Anti-bile duct cancer effect & $\downarrow N Q O 1$ & In vitro and in vivo & {$[53,54]$} \\
\hline \multirow[t]{2}{*}{$\beta$-Sitosterol } & Anti-melanoma effect & $\downarrow J N K$ & In vitro & {$[77]$} \\
\hline & Anti-gastric cancer effect & 个PTEN/p-AMPK & In vitro and in vivo & [78] \\
\hline
\end{tabular}

acetic-acid writhing test, surgical incision, partial sciatic nerve ligation and complete Freund's adjuvant. Further study exerted that naloxone could not block the antinociceptive effect of stigmasterol [74]. Recent study indicated that stigmasterol exhibited anti-diabetic activity in vitro and in vivo. Stigmasterol could increase GLUT4 translocation and expression. In the animal model, stigmasterol could reduce insulin resistance and oral glucose tolerance with the decrease of fasting blood-glucose levels and blood lipid indexes [75]. Recently, stigmasterol and $\beta$-sitosterol could suppress colitis in dextran sulfate sodium (DSS)-induced colitis in C57BL/6J male mice fed a high fat Western-style diet. $\beta$-Sitosterol and stigmasterol critically suppressed colon shortening, decreased fecal hemoglobin content, and inhibited the severity of colitis in the middle as well as distal colon through the inactivation of NF- $\mathrm{kB}$. Particularly, stigmasterol could reduce COX-2 and CSF-1 [76].

\section{$\beta$-Sitosterol}

$\beta$-Sitosterol is a phytosterol, and could be extracted from Nigella sativa, Serenoa repens, Cucurbita pepo, Pygeum africanum, AL and etc. The melting point of $\beta$-sitosterol is $136-140{ }^{\circ} \mathrm{C}$. $\beta$-Sitosterol displayed cytotoxic effect on cancer cells and an anti-inflammatory activity. Multiple myeloma refers to a cancer of plasma cells. Recently, it was reported that $\beta$-sitosterol could display cytotoxicity on multiple myeloma U266 cells through apoptosis. Further study showed that $\beta$-sitosterol could increase production, activate AMP-activated protein kinase (AMPK) acetyl-CoA carboxylase (ACC) and JNK pathways, with a decrease of p-AKT, S6K and vascular VEGF [77]. Gastric cancer refers to the cancer starting from stomach. Recently, $\beta$-sitosterol exerted the anti-cancer effect on AGS human gastric adenocarcinoma cells through apoptosis. Further study showed that $\beta$-sitosterol could increase phosphatase and tensin homolog (PTEN) and $\mathrm{p}$-AMPK expression, and decrease Hsp90 protein.
Moreover, $\beta$-sitosterol could inhibit tumor weight and volume according in AGS xenograft animal study [78]. Recent study showed that $\beta$-sitosterol exerted the antiinflammatory activity on rat model according to the rat paw edema test and the rat pleurisy assay [79]. A recent study manifested that $\beta$-sitosterol could ameliorate high fat diet induced dyslipidemia and hepatotoxicity in Swiss albino mice [80].

\section{Conclusion}

In recent time, herbs and extractions from TCM have been widely considered as the complementary and alternative medicine (CAM) for various diseases treatment [81, 82]. AL has been used as TCM for thousands of years, and current studies found that AL and some of its pure compounds exerted diverse biological activities. It is interesting to find that most of major components could exert cytotoxic effects on cancer cells, especially melanoma (Table 2). However, the animal studies are insufficient. Also, the effects of these drugs on normal cells and healthy animals are lacking. Meanwhile, it is worthwhile to explore the pharmacological activities of minor components identified in AL. Nevertheless, further studies to identify other pure compounds for drug development and novel pharmacological activities of AL are still necessary to facilitate research and development.

\section{Abbreviations}

ACC: acetyl-CoA carboxylase; AL: Atractylodes lancea Thunb. DC; AMPK: AMP-activated protein kinase; ASNA: adrenal sympathetic nerve activity; ATL-I: atractylenolide I; ATL-II: atractylenolide II; ATL-III: atractylenolide III; Atr: atractylodin; bFGF: basic fibroblast growth factor; $\left[\mathrm{Ca}^{2+}\right]$ : $\mathrm{Ca}^{2+}$ concentration; CAM: complementary and alternative medicine; CCA: cholangiocarcinoma; CP: constipation-prominent; CREB: CAMP response element binding protein; COX2: cyclooxygenase-2; DP: diarrheaprominent; DSS: dextran sulfate sodium; EGF: epidermal growth factor; G-CSF: granulocyte-colony stimulating factor; HUVEC: human umbilical vein endothelial cells; 5 -HT: 5-hydroxytryptamine; IAV: influenza A virus; IFN- $\beta$ : tumor necrosis factor receptor-associated factor 6 interferon- $\beta$; IL-8: interleukin-8; JNK: c-Jun N-terminal kinase; KPS: Karnofsky performance status; LPS: lipopolysaccharide; MAPK: mitogen-activated protein kinase; MCP: monocyte chemoattractant protein; MPO: myeloperoxidase; 
nAChR: nicotinic acetylcholine receptor; NO: nitric oxide; NOD: nucleotidebinding domain; NQO1: NAD(P)H-quinone oxidoreductase 1; OV: opisthorchis viverrini; OVA: ovalbumin; OVA slgE: OVA-specific immunoglobulin E; PE: petroleum ether; PET-CT: positron emission tomography-computed tomography; PGE2: prostaglandin E2; PIF: proteolysis-inducing factor; PMACl: phorbol 12-myristate 13-acetate and calcium ionophore A23187; PMBA: phenylenepolymethylene-bis-ammonium; PTEN: phosphatase and tensin homolog; SuCh: succinylcholine; TFF2: trefoil factor2; TLR4: toll like receptor 4; TNF-a: tumor necrosis factor-a; UGT: uridine 5'-iphospho-glucuronosyltransferases; VCAM-1: vascular cell adhesion molecule-1; VEGF: vascular endothelial growth factor.

\section{Authors' contributions}

$\mathrm{XJ}$ and PF drafted the manuscript. YL drew Fig. 1. PC designed and revised the manuscript. All authors read and approved the final manuscript.

\section{Author details}

${ }^{1}$ Chengdu University of Traditional Chinese Medicine, Chengdu, China. ${ }^{2}$ West China School of Pharmacy, Sichuan University, Chengdu, China.

\section{Acknowledgements}

Not applicable.

\section{Competing interests}

The authors declare that they have no competing interests.

\section{Availability of data and materials}

Not applicable.

\section{Consent for publication}

All of authors consent to publication of this work in Chinese Medicine.

\section{Ethics approval and consent to participate}

Not applicable.

\section{Funding}

National Natural Science Foundation of China No. 81630101 supported this work.

\section{Publisher's Note}

Springer Nature remains neutral with regard to jurisdictional claims in published maps and institutional affiliations.

\section{Received: 14 August 2018 Accepted: 20 November 2018}

Published online: 27 November 2018

\section{References}

1. Qian S, Wang L, Duan J, Feng $H$. The research progress in chemical constituents and biological activities of Atractylodes lancea DC. Chin Tradit Herbal Drugs. 2006;18:8-11.

2. Koonrungsesomboon N, Nabangchang K, Karbwang J. Therapeutic potential and pharmacological activities of Atractylodes lancea (Thunb.) DC. Asian Pac. J Trop Med. 2014;7:421.

3. Zhang Y, Wang Z, Zhu J, Chen B, Li Y. Determination of atractylodin in rat plasma by HPLC-UV method and its application to a Pharmacokinetic study. J Liq Chromatogr R T. 2012;35:778-87.

4. Huo Y, Liu YQ, Bai ZX, Cai Q. Determination of (4E, 6E, 12E)-tetradecatriene-8,10-diyne-1,3-diyl diacetate in rat plasma and tissues by HPLC-UV method and their application to a pharmacokinetic and tissue distribution study. J Anal Methods Chem Biol. 2014;2014:1-7.

5. Liu Q, Kong D, Luo J, Kong W, Guo W, Yang M. Quantitative and fingerprinting analysis of Atractylodes rhizome based on gas chromatography with flame ionization detection combined with chemometrics. J Sep Sci. 2016;39:2517.

6. Duan JA, Wang L, Qian S, Su S. A new cytotoxic prenylated dihydrobenzofuran derivative and other chemical constituents from the rhizomes of Atractylodes lancea DC. Arch Pharm Res. 2008;31:965-9.
7. Ohara K, Katayama M, Nagai K. $\beta$-eudesmol, an oxygenized sesquiterpene, affects efferent adrenal sympathetic nerve activity via transient receptor potential ankyrin 1 in rats. Neurosci Lett. 2018;684:18-24.

8. Kimura Y, Sumiyoshi M. Effects of an Atractylodes lancea rhizome extract and a volatile component $\beta$-eudesmol on gastrointestinal motility in mice. J Ethnopharmacol. 2012;141:530-6.

9. Yu Y, Jia TZ, Cai Q, Jiang N, Ma MY, Min DY, Yuan Y. Comparison of the antiulcer activity between the crude and bran-processed Atractylodes lancea in the rat model of gastric ulcer induced by acetic acid. J Ethnopharmacol. 2015;160:211-8.

10. Wang M, Chen X. Obstacle and countermeasure of sustainable high yield for peanut in low-hilly red soil region. J Peanut Sci. 2005;34:17-22.

11. Mitsuboshi M, Kioka Y, Noguchi K, Asakawa S. Evaluation of suppressiveness of soils exhibiting soil-borne disease suppression after long-term application of organic amendments by the co-cultivation method of pathogenic fusarium oxysporum and indigenous soil microorganisms. Microbes Environ. 2018;33:58-65.

12. Zhao $M$, Wang $Q$, Ouyang $Z$, Han B, Wang W, Wei $Y$, Wu Y, Yang B. Selective fraction of Atractylodes lancea (Thunb.) DC. and its growth inhibitory effect on human gastric cancer cells. Cytotechnology. 2014:66:201-8.

13. Shimato Y, Ota M, Asai K, Atsumi T, Tabuchi Y, Makino T. Comparison of byakujutsu (Atractylodes rhizome) and sojutsu (Atractylodes lancea rhizome) on anti-inflammatory and immunostimulative effects in vitro. J Nat Med. 2018;72:192-201.

14. Jiao P, Tseng-Crank J, Corneliusen B, Yimam M, Hodges M, Hong M, Maurseth C, Oh M, Kim H, Chu M, Jia Q. Lipase inhibition and antiobesity effect of Atractylodes lancea. Planta Med. 2014;80:577-82.

15. Murayama C, Wang CC, Michihara S, Norimoto H. Pharmacological effects of "jutsu" (Atractylodis rhizome and Atractylodis lanceae rhizome) on 1-(2,5-dimethoxy-4-iodophenyl)-2-aminopropane (DOI)-induced head twitch response in mice (I). Molecules. 2014;19:14979-86.

16. Wei Y, Sui DJ, Xu HM, Ouyang Z, Wu N, Wang DJ, Zhang XY, Qian DW. Atractylodes lancea rhizome water extract reduces triptolide-induced toxicity and enhances anti-inflammatory effects. Chin J Nat Med. 2017:15:905-11.

17. Yu R, Yu BX, Chen JF, Lv XY, Yan ZJ, Cheng Y, Ma Q. Anti-tumor effects of Atractylenolide I on bladder cancer cells. J Exp Clin Cana Res. 2016;35:40.

18. Wang CC, Lin SY, Cheng CH, Hou WC. Pro-oxidant and cytotoxic activities of atractylenolide I in human promyeloleukemic HL-60 cells. Food Chem Toxicol. 2006;44:1308-15.

19. Wang CH, Duan HJ, He LC. Inhibitory effect of atractylenolide I on angiogenesis in chronic inflammation in vivo and in vitro. Eur J Pharmacol. 2002;612:143-52

20. Lim H, Lee JH, Kim J, Kim YS, Kim HP. Effects of the rhizomes of Atractylodes japonica and atractylenolide I on allergic response and experimental atopic dermatitis. Arch Pharm Res. 2004;35:2007-12.

21. Wang C, He L, Wang N, Liu F. Screening anti-inflammatory components from chinese traditional medicines using a peritoneal macrophage/cell membrane chromatography-offline-gc/ms method. J Chromatogr B Analyt Technol Biomed Life Sci. 2009;877:3019-24.

22. Carneiro BA, Meeks JJ, Kuzel TM, Scaranti M, Abdulkadir SA, Giles FJ. Emerging therapeutic targets in bladder cancer. Cancer Treat Rev. 2015;41:170-8.

23. Huang HL, Lin TW, Huang YL, Huang RL. Induction of apoptosis and differentiation by atractylenolide-1 isolated from Atractylodes macrocephala in human leukemia cells. Bioorg Med Chem Lett. 2016;26:1905-9.

24. Tentori L, Lacal PM, Graziani G. Challenging resistance mechanisms to therapies for metastatic melanoma. Trends Pharmacol Sci Rep. 2013;34:656-66.

25. Fu XQ, Chou JY, Li T, Zhu PL, Li JK, Yin CL, Su T, Guo H, Lee KW, Hossen MJ, Chou GX, YU ZL. The JAK2/STAT3 pathway is involved in the anti-melanoma effects of atractylenolide I. Exp Dermatol. 2018;27:201-4.

26. Liu Y, Jia Z, Dong L, Wang R, Qiu G. A randomized pilot study of atractylenolide I on gastric cancer cachexia patients. Evid-Based Comp Alt. 2008:5:337-44.

27. Song HP, Hou XQ, Li RY, Yu R, Li X, Zhou SN, Huang HY, Cai X, Zhou C. Atractylenolide I stimulates intestinal epithelial repair through polyamine-mediated $\mathrm{Ca}(2+)$ signaling pathway. Phytomedicine. 2017:28:27-35.

28. Li W, Zhi W, Liu F, He Z, Wang X, Niu X. Atractylenolide I restores HO-1 expression and inhibits OX-LDL-induced VSMCs proliferation, migration and inflammatory responses in vitro. Exp Cell Res. 2017;353:26-34. 
29. Zhang JL, Huang WM, Zeng QY. Atractylenolide I protects mice from lipopolysaccharide-induced acute lung injury. Eur J Pharmacol. 2015;765:94-9.

30. Zhang Q, Cao YF, Ran RX, Li RS, Wu X, Dong PP, Zhang YY, Hu CM, Wang WM. Strong specific inhibition of UDP-glucuronosyltransferase 2B7 by atractylenolide I and III. Phytother Res. 2016;30:25-30.

31. Lim H, Lee JH, Kim J, Kim YS, Kim HP. Effects of the rhizomes of Atractylodes japonica and atractylenolide I on allergic response and experimental atopic dermatitis. Arch Pharm Res. 2012;35:2007-12.

32. Resch M, Steigel A, Chen ZL, Bauer R. 5-Lipoxygenase and cyclooxygenase-1 inhibitory active compounds from Atractylodes lancea. J Nat Prod. 1998;61:347-50

33. Fu XQ, Chou GX, Kwan HY, Tse AK, Zhao LH, Yuen TK, Cao HH, Yu H, Chao XJ, Su T, Cheng BC, Sun XG, Yu ZL. Inhibition of STAT3 signalling contributes to the antimelanoma action of atractylenolide II. Exp Dermatol. 2014;23:855-7.

34. Tian S, Yu H. Atractylenolide II inhibits proliferation, motility and induces apoptosis in human gastric carcinoma cell lines HGC-27 and AGS. Molecules. 2017;22:1886.

35. Wang CC, Chen LG, Yang LL. Cytotoxic activity of sesquiterpenoids from Atractylodes ovata on leukemia cell lines. Planta Med. 2002;68:204-8.

36. Li CQ, He LC, Jin JQ. Atractylenolide I and atractylenolide III inhibit lipopolysaccharide-induced TNF-a and NO production in macrophages. Phytother Res. 2007;21:347-53.

37. Wang HX, Liu CM, Liu Q, Gao K. Three types of sesquiterpenes from rhizomes of Atractylodes lancea. Phytochemistry. 2008;69:2088-94.

38. Theoharides TC, Cochrane DE. Critical role of mast cells in inflammatory diseases and the effect of acute stress. J Neuroimmunol. 2004;146:1-12.

39. Yoou MS, Nam SY, Jin MH, Lee SY, Kim MS, Roh SS, Choi IH, Woo N, Lim S, Kim DH, Jang JB, Kim HM, Jeong HJ. Ameliorative effect of atractylenolide III in the mast cell proliferation induced by TSLP. Food Chem Toxicol. 2017;106:78-85.

40. Ji GQ, Chen RQ, Wang L. Anti-inflammatory activity of atractylenolide II through inhibition of nuclear factor-kappaB and mitogen-activated protein kinase pathways in mouse macrophages. Immunopharm Immunot. 2016;38:98-102.

41. Zhao H, Ji ZH, Liu C, Yu XY. Neuroprotection and mechanisms of atractylenolide III in preventing learning and memory impairment induced by chronic high-dose homocysteine administration in rats. Neuroscience. 2015;290:485-91.

42. Hwang JM, Tseng TH, Hsieh YS, Chou FP, Wang CJ, Chu CY. Inhibitory effect of atractylon on tert-butyl hydroperoxide induced DNA damage and hepatic toxicity in rat hepatocytes. Arch Toxicol. 2013;70:660-4.

43. Sin KS, Kim HP, Lee WC, Pachaly P. Pharmacological activities of the constituents of Atractylodes rhizomes. Arch Pharm Res. 1989;12:236-8.

44. Kiso Y, Tohkin M, Hikino H. Mechanism of antihepatotoxic activity of atractylon, I: effect on free radical generation and lipid peroxidation. Planta Med. 2015;51:97-100.

45. Guo FQ, Huang LF, Zhou SY, Zhang TM, Liang YZ. Comparison of the volatile compounds of Atractylodes, medicinal plants by headspace solid-phase microextraction-gas chromatography-mass spectrometry. Anal Chim Acta. 2006;570:73-8.

46. Kim HY, Nam SY, Hwang SY, Kim HM, Jeong HJ. Atractylone, an active constituent of KMP6, attenuates allergic inflammation on allergic rhinitis in vitro and in vivo models. Mol Immunol. 2016;78:121-32.

47. Cheng Y, Mai JY, Hou TL, Ping J, Chen JJ. Antiviral activities of atractylon from Atractylodis Rhizoma. Mol Med Rep. 2016;14:3704-10.

48. Han NR, Moon PD, Nam SY, Ryu KJ, Yoou MS, Choi JH, Hwang SY, Kim HM, Jeong HJ. Inhibitory effects of atractylone on mast cell-mediated allergic reactions. Chem-biol Interact. 2016;258:59-68.

49. Nakai Y, Kido T, Hashimoto K, Kase Y, Sakakibara I, Higuchi M, Sasaki H. Effect of the rhizomes of Atractylodes lancea and its constituents on the delay of gastric emptying. J Ethnopharmacol. 2003;84:51-5.

50. Masuda Y, Kadokura T, Ishii M, Takada K, Kitajima J. Hinesol, a compound isolated from the essential oils of Atractylodes lancea rhizome, inhibits cell growth and induces apoptosis in human leukemia HL-60 cells. J Nat Med. 2015;69:332-9.

51. Satoh K, Nagai F, Kano I. Inhibition of H+, K+-ATPase by hinesol, a major component of So-jutsu, by interaction with enzyme in the E1 state. Biochem Pharmacol. 2000;59:881-6.
52. Ben Sghaier M, Mousslim M, Pagano A, Ammari Y, Luis J, Kovacic H. beta-eudesmol, a sesquiterpene from Teucrium ramosissimum, inhibits superoxide production, proliferation, adhesion and migration of human tumor cell. Environ Toxicol Pharm. 2016;46:227-33.

53. Srijiwangsa P, Ponnikorn S, Na-Bangchang K. Effect of of vacic $\mathrm{H}$. betaeusuppression-enhanced sensitivity of cholangiocarcinoma cells to chemotherapeutic agents. BMC. Pharmacol Toxicol. 2018;19:32.

54. Plengsuriyakarn T, Karbwang J, Na-Bangchang K. Anticancer activity using positron emission tomography-computed tomography and pharmacokinetics of beta-eudesmol in human cholangiocarcinoma xenografted nude mouse model. Clin Exp Pharmacol. 2015;42:293-304

55. Kotawong K, Chaijaroenkul W, Muhamad P, Na-Bangchang K. Cytotoxic activities and effects of atractylodin and $\beta$-eudesmol on the cell cycle arrest and apoptosis on cholangiocarcinoma cell line. J Pharmacol Sci. 2018;136:51-6.

56. Ferlay J, Soerjomataram I, Dikshit R, Eser S, Mathers C, Rebelo M, Parkin DM, Forman D, Bray F. Cancer incidence and mortality worldwide: sources, methods and major patterns in GLOBOCAN 2012. Int I Cancer. 2015;136:E359-86.

57. Bomfim DS, Ferraz RP, Carvalho NC, Soares MB, Pinheiro ML, Costa EV, Bezerra DP. Eudesmol isomers induce caspase-mediated apoptosis in human hepatocellular carcinoma HepG2 cells. Basic Clin Pharmacol. 2013;113:300-6

58. Tsuneki H, Ma EL, Kobayashi S, Sekizaki N, Maekawa K, Sasaoka T, Wang MW, Kimura I. Antiangiogenic activity of beta-eudesmol in vitro and in vivo. Eur J Pharmacol. 2005;512:105-15.

59. Ma EL, Li YC, Tsuneki H, Xiao JF, Xia MY, Wang MW, Kimura I. Betaeudesmol suppresses tumour growth through inhibition of tumour neovascularisation and tumour cell proliferation. J Asian Nat Prod Res. 2008;10:159-67.

60. Obara Y, Aoki T, Kusano M, Ohizumi Y. Beta-eudesmol induces neurite outgrowth in rat pheochromocytoma cells accompanied by an activation of mitogen-activated protein kinase. J Pharmacol Exp Ther. 2002;301:803-11.

61. Muroi M, Tanaka K, Kimura I, Kimura M. Beta-eudesmol (a main component of Atractylodes lancea)-induced potentiation of depolarizing neuromuscular blockade in diaphragm muscles of normal and diabetic mice. Jpn J Pharmacol. 1989;50:69-71.

62. Nojima H, Kimura I, Kimura M. Blocking action of succinylcholine with beta-eudesmol on acetylcholine-activated channel activity at endplates of single muscle cells of adult mice. Brain Res. 1992;575:337-40.

63. Kimura M, Kimura I, Muroi M, Tanaka K, Nojima H, Uwano T. Different modes of potentiation by beta-eudesmol, a main compound from Atractylodes lancea, depending on neuromuscular blocking actions of p-phenylene-polymethylene bis-ammonium derivatives in isolated phrenic nerve-diaphragm muscles of normal and alloxan-diabetic mice. Jpn J Pharmacol. 1992;60:19-24.

64. Marinho CG, Della Lucia TM, Ribeiro MM, Magalhaes ST, Guedes RN, Jham GN. Interference of beta-eudesmol in nestmate recognition in Atta sexdens rubropilosa (Hymenoptera: Formicidae). B Entomol Res. 2008;98:467-73.

65. Kimura I, Tsuneki H, Kondoh T, Kimura M. Enhancing effect by nicotinic acetylcholine receptor channel blockers, including beta-eudesmol, on succinylcholine-induced inhibition of twitch tension and intracellular $\mathrm{Ca}^{2+}$ in mouse diaphragm muscle. J Pharmacol Exp Ther. 1991;256:24-8

66. Tang F, Fan K, Wang K, Bian C. Atractylodin attenuates lipopolysaccharide-induced acute lung injury by inhibiting NLRP3 inflammasome and TLR4 pathways. J Pharmacol Sci. 2018;136:203-11.

67. Yu C, Xiong Y, Chen D, Li Y, Xu B, Lin Y, Tang Z, Jiang C, Wang L. Ameliorative effects of atractylodin on intestinal inflammation and co-occurring dysmotility in both constipation and diarrhea prominent rats. Korean J Physiol Pha. 2017;21:1-9.

68. Antwi AO, Obiri DD, Osafo N. Stigmasterol modulates allergic airway inflammation in guinea pig model of ovalbumin-induced asthma. Mediat Inflamm. 2017;1:1. https://doi.org/10.1155/2017/2953930.

69. Adebiyi OE, Olopade JO, Olayemi FO. Sodium metavanadate induced cognitive decline, behavioral impairments, oxidative stress and down regulation of myelin basic protein in mice hippocampus: ameliorative roles of $\beta$-spinasterol, and stigmasterol. Brain Behav. 2018;8:e01014. 
70. Antwi AO, Obiri DD, Osafo N, Forkuo AD, Essel LB. Stigmasterol inhibits lipopolysaccharide-induced innate immune responses in murine models. Int Immunopharmacol. 2017:53:105-13.

71. Yenn TW, Arslan Khan M, Amiera Syuhada N, Chean Ring L, Ibrahim D, Tan WN. Stigmasterol: an adjuvant for beta lactam antibiotics against betalactamase positive clinical isolates. Steroids. 2017;128:68-71.

72. Aminu R, Umar IA, Rahman MA, Ibrahim MA. Stigmasterol retards the proliferation and pathological features of Trypanosoma congolense infection in rats and inhibits trypanosomal sialidase in vitro and in silico. Biomed Pharmacother. 2017;89:482-9.

73. Gade S, Rajamanikyam M, Vadlapudi V, Nukala KM, Aluvala R, Giddigari C, Karanam NJ, Barua NC, Pandey R, Upadhyayula VSV, Sripadi P, Amanchy R, Upadhyayula SM. Acetylcholinesterase inhibitory activity of stigmasterol \& hexacosanol is responsible for larvicidal and repellent properties of Chromolaena odorata. BBA-Gen Subjects. 2017;1861:541-50.

74. Walker CIB, Oliveira SM, Tonello R, Rossato MF, da Silva Brum E, Ferreira J, Trevisan G. Anti-nociceptive effect of stigmasterol in mouse models of acute and chronic pain. N-S Arch Pharmacol. 2017;390:1163-72.

75. Wang J, Huang M, Yang J, Ma X, Zheng S, Deng S, Huang Y, Yang X, Zhao P. Anti-diabetic activity of stigmasterol from soybean oil by targeting the GLUT4 glucose transporter. Food Nutr Res. 2017;61:1364117.

76. Feng S, Dai Z, Liu A, Wang H, Chen J, Luo Z, Yang CS. $\beta$-Sitosterol and stigmasterol ameliorate dextran sulfate sodium-induced colitis in mice fed a high fat Western-style diet. Food Funct. 2017;8:4179-86.
77. Sook SH, Lee HJ, Kim JH, Sohn EJ, Jung JH, Kim B, Kim JH, Jeong SJ, Kim $\mathrm{SH}$. Reactive oxygen species-mediated activation of AMP-activated protein kinase and c-Jun $\mathrm{N}$-terminal kinase plays a critical role in betasitosterol-induced apoptosis in multiple myeloma U266 cells. Phytother Res. 2014;28:387-94.

78. Shin EJ, Choi HK, Sung MJ, Park JH, Chung MY, Chung S, Hwang JT. Antitumour effects of beta-sitosterol are mediated by AMPK/PTEN/HSP90 axis in AGS human gastric adenocarcinoma cells and xenograft mouse models. Biochem Pharmacol. 2018;152:60-70.

79. Paniagua-Pérez R, Flores-Mondragón G, Reyes-Legorreta C, Herrera-López B, Cervantes-Hernández I, Madrigal-Santillán O, Morales-González JA Álvarez-González I, Madrigal-Bujaidar E. Evaluation of the anti-inflammatory capacity of beta-sitosterol in rodent assays. Afr J Tradit Complement Altern Med. 2017;14:123-30.

80. Sikder K, Das N, Kesh SB, Dey S. Quercetin and beta-sitosterol prevent high fat diet induced dyslipidemia and hepatotoxicity in Swiss albino mice. Indian J Exp Biol. 2014;52:60-6.

81. Lewith G, Britten N, Paterson C. Complementary and alternative medicine (CAM) and cancer: the kind face of complementary medicine. Int J Surg. 2010;8:81.

82. Hsiao WL, Liu L. The role of traditional Chinese herbal medicines in cancer therapy-from TCM theory to mechanistic insights. Planta Med. 2010;76:1118-31.
Ready to submit your research? Choose BMC and benefit from:

- fast, convenient online submission

- thorough peer review by experienced researchers in your field

- rapid publication on acceptance

- support for research data, including large and complex data types

- gold Open Access which fosters wider collaboration and increased citations

- maximum visibility for your research: over 100M website views per year

At BMC, research is always in progress.

Learn more biomedcentral.com/submissions 